\title{
Socio-Demographic Factors and Epidemiological Characteristics of HIV-Positive Pregnant Women with High Risk of Vertical Transmission of the Immunodeficiency Virus
}

\author{
Anastasiya S. Vanyarkina*, $\mathrm{PhD}$; Alla G. Petrova, $\mathrm{PhD}, \mathrm{ScD}$; \\ Lyubov V. Rychkova, PhD, ScD; Ekaterina V. Moskaleva, PhD; Evgeniya A. Novikova \\ Scientific Centre for Family Health and Human Reproduction Problems \\ Irkutsk, the Russian Federation
}

\begin{abstract}
The purpose of this study was to determine the features of the course of pregnancy, delivery, and the postpartum period in HIV-positive women with a high risk of HIV vertical transmission.

Methods and Results: A retrospective, longitudinal cohort study of mother-child pairs for the period from 2017 to 2019 was conducted in the Irkutsk City Perinatal Center (level III). The clinical observation group included HIV-positive women (n=213) and their newborn children with a high risk of perinatal immunodeficiency virus transmission $(n=214)$. The findings of the conducted study demonstrated that most HIV-seropositive women with a high risk of HIV vertical transmission had an aggravated social history, a high prevalence of pelvic inflammatory diseases, and a high incidence of opportunistic and AIDS-defining conditions. Evaluation of PMTCT preventive complex showed that the target parameters in women with a high risk of HIV transmission were not reached: the first stage was performed for $49.3 \%$ of pregnant women with good ART adherence, the second stage - for $97.1 \%$ of obstetric patients, the third stage - in 100\% of HIV perinatally exposed children. HIV RNA was detected in 3.7\% of children, which evidences their antenatal infection.

Conclusion: Development of efficient communication with HIV-positive women aimed at preservation of their health and decrease of logistic barriers to access to medical care.(International Journal of Biomedicine. 2021;11(4):564-569.)
\end{abstract}

Key Words: HIV infection • pregnancy outcomes • vertical transmission • retrospective study

For citation:Vanyarkina AS, Petrova AG, Rychkova LV, Moskaleva EV, Novikova EA. Socio-Demographic Factors and Epidemiological Characteristics of HIV-Positive Pregnant Women with High Risk of Vertical Transmission of the Immunodeficiency Virus. International Journal of Biomedicine. 2021;11(4):564-569. doi:10.21103/Article11(4)_OA27

\section{Abbreviations}

ART, antiretroviral therapy; HIV, human immunodeficiency virus; PMTCT, prevention of mother-to-child transmission; VL, viral load.

\section{Introduction}

The infection caused by human the immunodeficiency virus (HIV) continues to be a priority medical and social problem and seriously threatens the health of women and children. Significant progress in strategies aimed at preventing mother-to-child transmission (PMTCT) of HIV was achieved over the past decades; these strategies allow an HIV-positive woman to realize her reproductive potential.
However, the final elimination of HIV infection in the pediatric population directly depends on parental HIV status, which actualizes the study of the nature of disease distribution and necessitates taking coordinated tactical decisions. HIV infection continues to be registered in all the constituents of the Russian Federation, and the number of constituent regions with a high prevalence of the disease is continuously growing (more than $0.5 \%$ of the overall population number): from 22 in 2014 to 38 in 2020 . One of the territories is the Irkutsk region, 
where 1,951.5 HIV-infected people have been registered per 100,000 of the population since 2020 . The high prevalence of HIV in the region is also among fertile women. ${ }^{(1,2)}$

Despite the trend to decrease the incidence of HIV vertical transmission, we should not forget about the abnormal reproductive health of an HIV-positive woman, which can not only complicate the course of pregnancy and delivery, but also affect the state of a newborn. ${ }^{(1)}$ It should be noted that an HIV-positive woman often has comorbid and severe forms of the disease which significantly increases the incidence of unfavorable perinatal outcomes. ${ }^{(2-4)}$ Positive epidemiological anamnesis and decreased access of HIV-positive fertile women to specialty care also increase the probability of mother-tochild transmission of HIV because the target parameters of three-stage chemoprophylaxis of vertical transmission of HIV infection (during pregnancy, delivery, and to a newborn) are still unachieved.

Therefore, further in-depth study to understand the features of perinatal outcomes in HIV-infected women who have maximum risks of mother-to-child transmission of HIV and who are living in a territory with a high prevalence. It is a necessary condition to successfully realize a regional, adopted system of activities to control the HIV infection epidemic and completely eliminate mother-to-child transmission of the disease.

The purpose of this study was to determine the features of the course of pregnancy, delivery, and the postpartum period in HIV-positive women with a high risk of HIV vertical transmission.

\section{Materials and Methods}

A retrospective, longitudinal cohort study of motherchild pairs for the period from 2017 to 2019 was conducted in the Irkutsk City Perinatal Center (level III). The clinical observation group included HIV-positive women $(n=213)$ and their newborn children with a high risk of perinatal immunodeficiency virus transmission $(\mathrm{n}=214)$.

The inclusion criteria were: no maternal chemoprophylaxis during pregnancy within at least four previous weeks; no chemoprophylaxis during pregnancy and/ or delivery; insufficient pre-delivery prophylaxis ( $\mathrm{VL}>50$ copies/ml prior to delivery); positive maternal rapid HIV test at delivery; the increased risk of infection during pregnancy (epidemiological indications, i.e. injecting a drug within the previous 12 weeks or sexual contact with an HIV-positive partner).

Since birth, all the HIV perinatally exposed children received ART with three-drug products (zidovudine, nevirapine, lamivudine), formula-fed and tested for HIV nucleic acids by molecular and genetic techniques within the first 48 hours of life.

Several maternal factors affecting the state of a child after delivery were evaluated. External (social) maternal factors include low social status, the absence of a family and/or a partner, regular place of work, high/professional education; substance use during the current pregnancy. Internal (medicinal) maternal factors include HIV-positive status; coinfections (hepatitis C and/or B virus, herpes virus infections, fungal infections); HIV-VL at pregnancy; compliance with ART at pregnancy (as part of PMTCT of HIV as well as treatment of maternal HIV infection prior to pregnancy). Medical records of each HIV-positive woman and her child were reviewed; clinical observation and examination of children within the specified group for their first 28 days of lifee, and their microbiological and virological monitoring were performed.

Statistical analysis was performed using the Statistica 6.1 software package (Stat-Soft Inc., USA). For descriptive analysis, results are presented as median (Me) and interquartile range (IQR; 25th to 75th percentiles). The frequencies of categorical variables were compared using Pearson's chisquared test. The Wilcoxon signed-rank test was used to compare the differences between the two dependent groups (for non-parametric data). Odds ratios (ORs) and their 95\% confidence intervals (CIs) were calculated A probability value of $P<0.05$ was considered statistically significant.

\section{Results}

The study findings showed that the median and quartiles of the age of pregnant women with a high risk of HIV vertical transmission were 30(27-34 years). Table 1 demonstrates a roughly equal ratio of female patients in the age categories from 21 to 30 years of age ( $46 \%$ ) and from 31 to 40 years of age (45\%). The majority of HIV-positive pregnant women were Russians $(97.6 \%)$, permanently residing in the city $(71.8 \%)$, and had secondary $(38 \%)$ or lower secondary $(30.9 \%)$ education. Interestingly, $74.2 \%$ of HIV-positive women were found not to be active in the labor force before they became pregnant. By social position, $1.8 \%$ of the unemployed HIV-seropositive pregnant women were students, $98.2 \%$ - housewives; $63.3 \%$ of HIV-positive women were not in a registered marriage. The partners of one-third of pregnant women $(31.45 \%)$ were also HIV-seropositive, and the percentage of women with the annulled parental rights was $7 \%$.

The study findings showed that $80 \%$ of women had a history of substance use. Smoking cigarettes and substance use were found to be most common among HIV-infected women $(56.8 \%$ and $13.6 \%$ cases, respectively); combined substance use was observed often. Most women with a risk of HIV vertical transmission continued to inject drugs.

The evaluation of the obstetric and gynecological history of HIV-positive women with a high risk of HIV vertical transmission showed that pregnancy was planned by only one-third of female patients $(29.1 \%)$. The median and quartiles of pregnancy ranking were found to be $3(2-5)$, and the first pregnancy was recorded only in $10.3 \%$ of women. Social deprivation was also reflected at the antenatal phase of monitoring HIV-positive pregnant women. The median and the quartiles of pregnancy registration date were shown to be $13(9-20)$. About $78.8 \%$ of HIV-positive women were registered at the female health department. However, only half of them were registered prior to Week 12 of gestation $(54.7 \%)$, and irregular perinatal care was found in $60.7 \%$ of cases. 
Table 1.

Social and demographic characteristics of pregnant women with a high risk of perinatal HIV transmission $(n=213)$

\begin{tabular}{|c|c|c|}
\hline Parameter & Abs. & $\%[95 \% \mathrm{CI}]$ \\
\hline \multicolumn{3}{|c|}{ Age } \\
\hline$<20$ years & 17 & $7.98[4.8-12.6]$ \\
\hline $21-30$ years & 98 & $46[39.2-52.9]$ \\
\hline $31-40$ years & 96 & $45[38.3-52]$ \\
\hline$>40$ years & 3 & $1.4[0.37-4.4]$ \\
\hline \multicolumn{3}{|c|}{ Ethnicity } \\
\hline Russians & 208 & 97.65 [94.3-99.13] \\
\hline \multicolumn{3}{|c|}{ Place of residence } \\
\hline City & 153 & $71.8[65.2-77.66]$ \\
\hline \multicolumn{3}{|c|}{ Education } \\
\hline Lower secondary & 66 & 30.99 [24.9-37.7] \\
\hline Secondary & 81 & 38 [31.5-44.9] \\
\hline Secondary special & 44 & $20.66[15.5-26.8]$ \\
\hline High & 22 & $10.33[6.7-15.4]$ \\
\hline Primary school & 2 & $0.9[0.16-3.7]$ \\
\hline \multicolumn{3}{|l|}{ Other } \\
\hline Employment & 55 & $25.8[20.1-32.3]$ \\
\hline Marriage not registered & 135 & $63.3[56.4-69.7]$ \\
\hline HIV-positive partner & 67 & $31.4[25.3-38.2]$ \\
\hline Deprivation of paternal rights & 15 & $7[4,1-11,5]$ \\
\hline \multicolumn{3}{|c|}{ Substances use } \\
\hline Nicotine & 121 & $56.8[49.8-63.5]$ \\
\hline Alcohol & 21 & $9,8[6.3-14.8]$ \\
\hline Parenteral drugs & 29 & $13,6[9.4-19.1]$ \\
\hline
\end{tabular}

The obstetric history of many HIV-seropositive pregnant women was aggravated: $15 \%$ had a premature birth, $17.3 \%$ - miscarriages, and 48.3\% - induced abortions. Many were diagnosed with chronic inflammatory diseases of reproductive system organs: salpingitis $(1.8 \%)$, oophoritis $(0.4 \%)$, vaginitis $(2.8 \%)$, cervicitis $(8.4 \%)$, and colpitis $(17.8 \%)$.

Table 2 shows the structure of somatic pathology in HIV-positive women. The leading nosologies were urinary system inflammatory diseases (13.6\%) and endocrinopathies (5.6\%). Reproductive system tumors at different sites were not uncommon $(3.2 \%)$.

Interestingly, various abnormalities in the course of pregnancy were observed in most women with a high risk of HIV transmission. Change in the amount of amniotic fluid was observed in HIV-positive women equally as often hydramnios (1.4\%) and oligoamnions (1.4\%); $4.6 \%$ of patients were diagnosed with preeclampsia of various severities, $11.7 \%$ had threatened miscarriage, $26.7 \%$ - placental insufficiency, $3.7 \%$ - gestational diabetes. Anemia prevailed (87.7\%) among hematologic disorders in HIV-seropositive pregnant women; thrombocytopenia $(1.8 \%)$ and bleeding abnormality as hypercoagulation $(0.4 \%)$ were also observed.

According to our data, many women with a high risk of HIV vertical transmission at pregnancy and delivery had inflammatory diseases: gestational pyelonephritis $(2.8 \%)$, chorioamnionitis $(2.8 \%)$, cryptogenic hepatitis $(0.4 \%)$, community-acquired pneumonia (0.4\%), acute enteric infection, and ARVI (4.5\%). One $(0.4 \%)$ HIV-seropositive woman was diagnosed with sepsis. The evaluation of verified infectious pathology showed that hepatitis C (49.2\%), syphilis (23.9\%), mycotic infection (31.9\%) as well as pulmonary tuberculosis $(8.4 \%)$ were the dominant nosologies.

Table 2.

Somatic pathology in pregnant women with a high risk of perinatal HIV transmission $(n=213)$

\begin{tabular}{|l|c|c|}
\hline \multicolumn{1}{|c|}{ Somatic pathology } & Abs. & $\%[95 \% \mathrm{CI}]$ \\
\hline Respiratory tract diseases & 6 & $2.8[1.1-6.3]$ \\
\hline Cardiovascular system diseases & 2 & $0.9[0.16-3.7]$ \\
\hline Genitourinary system diseases & 29 & $13.6[9.4-19.1]$ \\
\hline Gastrointestinal tract diseases & 2 & $0.9[0.16-3.7]$ \\
\hline Neuropsychiatric diseases & 3 & $1.4[0.37-4.4]$ \\
\hline Endocrinopathies & 12 & $5.6[3-9.8]$ \\
\hline Reproductive system neoplasms & 7 & $3.2[1.4-6.9]$ \\
\hline $\begin{array}{l}\text { Inflammatory diseases of } \\
\text { reproductive system }\end{array}$ & 67 & $31.4[25.3-38.2]$ \\
\hline
\end{tabular}

The evaluation of the course of the intranatal period established that most pregnant women with a high risk of HIV vertical transmission were waiting for the second labor (83\%). About $1.4 \%$ of women had outpatient labor; the others were hospitalized for labor; $36.1 \%$ of HIV-positive women had premature labor, $17.3 \%-<12$-hour period between rupture and delivery, $4.2 \%$ - abnormal labor, $1.4 \%$ - a fetal presentation, $1.4 \%$ - umbilical cord prolapse. One HIV-seropositive woman had ingrowth of placenta to the uterine wall, $3.2 \%$ a complete detachment of a normally situated placenta, and $1.8 \%$ - hemorrhage at the fourth stage of labor with major blood loss. Some women had invasive procedures at delivery by urgent obstetric indications: $0.9 \%$ underwent amniotomy, $2.3 \%$ - episiotomy. One patient underwent a hysterectomy during the postpartum period due to major bleeding.

Careful examination of epidemiological anamnesis is performed for making a decision on the management of female patients admitted to an obstetric institution with a confirmed diagnosis of HIV infection or without perinatal care. Evaluation of HIV status of women with a high risk of vertical transmission of immunodeficiency virus established that most patients were diagnosed before the current pregnancy (70.4\%). One-third of patients became aware of their HIV-seropositive status when they sought medical attention during the current pregnancy $(29.5 \%)$. Median and quartiles of HIV infection duration were 3(1-7) years. The main route of transmission was heterosexual contacts $(61.5 \%)$ and the parenteral route $(12.6 \%)$. The HIV transmission route was unknown in $25.3 \%$ of cases. The study of HIV status showed that most women were diagnosed with subclinical Stage $3(36.1 \%)$ and secondary diseases Stages 4A, 4B, 4C(40.3\%).

Of mothers with HIV infection, $10.8 \%$ were found to be continuously monitored in the regional center for prevention 
and control of AIDS and received ART prior to pregnancy. Only $68.5 \%$ of patients with a current pregnancy visited an infectious disease specialist, and the monitoring was regular only in $69.8 \%$ of women in this cohort. Chemoprophylaxis regimens of HIV infection treatment (first stage of PMTCT) were administered to all the women at pregnancy when they visited an infectious disease specialist as per the protocols. However, their compliance with the treatment was not high; only $49.3 \%$ of HIV-positive women adhered to the medical prescriptions.

Pregnant women observed in the regional center for prevention and control of AIDS showed a 4.7-fold decrease in the VL at control examination on Weeks 34-36 of gestation, as compared to the first visit of an infectious disease specialist $(\mathrm{Z}=3.4 ; P \mathrm{~W}=0,0006)$. A statistically significant decrease of HIV-positive women with VL of 100,000 copies $/ \mathrm{ml}$ and more $(\chi 2=17,7 ; \mathrm{df}=1 ; P<0,001)$ (Table 3$)$ was observed.

\section{Table 3.}

Dynamics of the HIV viral load of the examined women with a high risk of HIV vertical transmission, observed at the Regional Center on Prevention and Control of AIDS $(n=146)$

\begin{tabular}{|l|c|c|c|c|c|}
\hline \multirow{2}{*}{$\begin{array}{l}\text { Viral load, } \\
\text { cop/ml }\end{array}$} & \multicolumn{4}{|c|}{$\begin{array}{c}\text { Observation stages } \\
\text { pregnancy }\end{array}$} & \multicolumn{2}{|c|}{$\begin{array}{c}\text { Visit at Weeks } \\
\text { prisit during of gestation }\end{array}$} & \\
\cline { 2 - 6 } & Abs & $\%[95 \%$-value & Abs & $\%[95 \% \mathrm{CI}]$ & \\
\hline$<400$ & 32 & $21.9[15.6-29.6]$ & 37 & $25.3[18.6-33.3]$ & 0.49 \\
\hline $400-10000$ & 49 & $33.5[26-41.9]$ & 43 & $29.4[22.3-37.6]$ & 0.44 \\
\hline$>10001$ & 61 & $41.7[33.7-50.2]$ & 47 & $18.4[12.7-25.9]^{*}$ & $<0.0001$ \\
\hline
\end{tabular}

*Statistically significant differences between the groups by $\chi^{2}$ criterion.

About $49 \%$ of pregnant women with adequate compliance with ART received chemoprophylaxis in the antenatal period, which was shown in the estimated adequacy of PMTCT preventive measures complex. The second stage of PMTCT was performed in $97.1 \%$ of obstetric patients with a high risk of HIV vertical transmission. No chemoprophylaxis of this stage in $2.8 \%$ of women was related to outpatient delivery or to admission in the active pushing phase. One hundred percent of newborns received ART and switched to formula feeding in the postnatal period.

The study of clinical and anamnestic features of HIV perinatally exposed newborns $(\mathrm{n}=214)$ found that more often children were born by cesarean section $(55.6 \%)$. The median body weight of these newborns was $2,630 \mathrm{~g}$ (IQR: $1980 \mathrm{~g}-3050 \mathrm{~g}$ ), median body height at birth was 46 cm (IQR: $28 \mathrm{~cm}-55 \mathrm{~cm}$ ), and median gestation age was 37 weeks(IQR: $34-38$ weeks). More than one-third of children were prematurely born $(35.9 \%), 15.5 \%$ - with extremely low body weight, $22 \%$ - very low body weight; $27.5 \%$ of newborns were small for gestation date; $3.7 \%$ had HIV RNA, which evidences their antenatal infection. The diagnosis was confirmed after examination of a second blood sample of a child taken on Day 2 after a positive result was received. All children were directed to a specialist on HIV infection for diagnosis verification, in-depth examination, and treatment administration.

\section{Discussion}

Socio-demographic factors of pregnant women with a high risk of HIV vertical transmission found in the study reflect regional and all-Russian trends. The influence of the educational level of women and their partners on the prevalence of HIV infection was previously shown by various authors. It was found that every additional year of education in an educational institution statistically significantly decreased the risk of HIV infection by $7 \%{ }^{(5)}$ It was also observed that better-educated women are capable of better processing the information on the infection risks, rules of safe behavior, and the need for preventive measures that mitigate the probability of HIV transmission and infection. ${ }^{(6)}$ Many authors previously noted a low behavioral and social status of pregnant HIVpositive women. ${ }^{(7-10)}$

The study showed that most women continued to inject drugs during pregnancy. This fact reflects regional features of the course of the epidemic process. It should be noted that the substances are an additional risk factor of the course of pregnancy in HIV-positive women and significantly affect the fetoplacental complex and a newborn's status. ${ }^{(11)}$ Smoking cigarettes during pregnancy is a proven risk factor for unfavorable outcomes in HIV-positive pregnant women, which is related to intrauterine fetal hypoxia, architectural distortion of placental vessels, inflammation, immune status abnormality, and increased risk of miscarriage..$^{(12-14)} \mathrm{It}$ should be noted that there is a causal relationship between HIV infection and the use of alcohol or drugs because such consumers are prone to risky behavior, which increases the risk of immunodeficiency virus infection. In addition, the response to HIV infection may be considered by a woman as a traumatic event that involves cognitive and behavioral as well as emotional aspects of mental health and needs psychosocial support. ${ }^{(15)}$

Our results showed a high prevalence of reproductive system diseases in women with a high risk of HIV vertical transmission. It should be stressed that a high prevalence of pelvic inflammatory diseases not only increases the probability of reproductive losses but also increases the risk of sexually transmitted diseases as well as activation of latent infections. ${ }^{(2,16,17)}$

The prevalence of kidney diseases in HIV-positive patients observed in our study was also noted by other authors. There is a probable relation between the pathogenetic role of HIV infection and the development of nephropathy. ${ }^{(8)}$ Literature data for the last years also show that not infrequently severe cervical injuries are recorded. ${ }^{(18)}$ High frequency of carriage of human papillomaviruses of oncogenic types in HIV-seropositive women as well as statistically significantly increased prevalence of cervical intraepithelial dysplasia were observed in the territory of Irkutsk city. ${ }^{(19)}$

As noted above, perinatal outcomes mainly depend on HIV infection comorbidity. The analysis of the findings again draws attention to the high prevalence of opportunistic 
infections and AIDS-defining conditions, which, on the one hand, may aggravate the immunosuppression and increase the risk of mother-to-child transmission of other pathogens, and on the other, HIV infection is considered an important determinant of hepatitis $\mathrm{C}$ virus vertical transmission from mother to child. Perinatal transmission of hepatitis $\mathrm{C}$ was demonstrated to be met in immunocompetent individuals $(<1 \%$ of cases), but it increases up to $20 \%$ in HIV-positive women with progressive immunodeficiency. The risk of perinatal transmission is supposed to be mitigated by the administration of ART during pregnancy and is less than $3 \%$ with operative delivery. ${ }^{(20)}$ It should be also stated that unbalanced oxidantantioxidant status is observed with HIV infection, which enables the sustained chronic inflammation and development of inadequate immune response. ${ }^{(21)}$

Our observations again emphasize the significance when patients comply with not only the therapy but also medical monitoring, which is one of the key parameters of health care delivery to HIV-positive patients. As is known, the leading factor affecting the probability of HIV transmission from mother to fetus or child is the blood concentration of the virus of a woman - VL during pregnancy and delivery. Despite the trend to decrease VL, the findings of our study show that most women with a high risk of HIV vertical transmission did not reach undetectable levels. The probability of motherto-child transmission is known to correlate with the VL and duration of antiretroviral therapy, meanwhile, about $75 \%$ of HIV transmission occurs during delivery or during the last few weeks of pregnancy. HIV vertical transmission prior to the third trimester of pregnancy is observed in about $10 \%$ of cases, during breastfeeding - in $10 \%-15 \%$. $^{(22)}$ If the VL in a woman is below the detection limit, the probability of vertical transmission is extremely low. On the other hand, absence or insufficient HIV suppression significantly reduces the risk of vertical transmission.

A vertical route of transmission was also observed in $3.7 \%$ of newborns. It was found that not one of the mothers of newborns with perinatal HIV transmission received ART during pregnancy. Most women were not registered at the female health department $(62.5 \%)$. A common use of substances during pregnancy was observed. HIV denialism was found in one woman, which interfered with antenatal and intranatal stages of PMTCT. The second stage of PMTCT was not implemented in $37.5 \%$ of HIV-positive women. The third stage of PMTCT at the postnatal stage (boosted ART regimen) was initiated in all newborns. However, the absence of a complete complex of preventive measures of PMTCT threatened the health of newborns and increased the probability of HIV vertical transmission, in spite of boosted ART drug products administered to all children of PMTCT postnatal stage.

\section{Conclusion}

The findings of the conducted study demonstrated that most HIV-seropositive women with a high risk of HIV vertical transmission had an aggravated social history, $80 \%$ used various substances, $74.2 \%$ were not active in the labor force, most $(60.7 \%)$ had no regular antenatal care during pregnancy. A high prevalence of pelvic inflammatory diseases(31\%), nephropathies(13.6\%), and endocrinopathies (5.6\%) was observed. High incidence of opportunistic and AIDS-defining conditions was recorded with hepatitis $\mathrm{C}(49.2 \%)$, syphilis(23.9\%), and fungal infection (31.9\%) prevailing. Abnormality of the intranatal period was observed in more than half of the women (65.7\%). The main routes of HIV transmission were heterosexual $(61.5 \%)$ and parenteral(12.6\%). Most women were diagnosed with HIV before their current pregnancy (70.5\%). Attention is paid to the low compliance of HIV-positive pregnant women with medical supervision and the use of ART. A 4.7-fold progressive decrease in VL was found in HIV-positive women who received specialty care $(\mathrm{Z}=3,4 ; \mathrm{pW}=0.0006)$. The number of pregnant women with a VL of 100,000 copies/ml and higher was also decreased $(\chi 2=17.7 ; \mathrm{df}=1 ; \mathrm{p}<0.001)$. However, an undetectable level was not reached due to ART use and low compliance of HIV-positive pregnant women with medical supervision, despite the availability of qualitative special medical care and the presence of efficient chemoprophylaxis regimens at pregnancy. Evaluation of PMTCT preventive complex showed that the target parameters in women with a high risk of HIV transmission were not reached: the first stage was performed for $49.3 \%$ of pregnant women with good ART adherence, the second stage - for $97.1 \%$ of obstetric patients, the third stage - in $100 \%$ of HIV perinatally exposed children. HIV RNA was detected in $3.7 \%$ of children, which evidences their antenatal infection.

Hence, the findings of our study show that each mother of a newborn in the high-risk group had anamnesis factors of pregnancy and delivery abnormalities, and a newborn had prerequisites for infection. This necessitates the enhancement of activities on medical and social support of women of fertile age from risk groups, on the provision of measures of psychological support with the transformation of their behavioral settings. Development of efficient communication with HIV-positive women aimed at preservation of their health and decrease of logistic barriers to access to medical care.

\section{Acknowledgments}

This article contains material that has been discussed at the VIII International Research and Practical Conference «FUNDAMENTAL AND APPLIED ASPECTS OF REPRODUCTION» (December 2021, Irkutsk, Russia). The author thanks all researchers who participated in the oral discussion.

\section{Competing Interests}

The authors declare that they have no competing interests.

*Corresponding author: Anastasiya Vanyarkina, PhD Scientific Centre for Family Health and Human Reproduction Problems, Irkutsk, the Russian Federation. E-mail. avanyarkina@ yahoo.com 


\section{References}

1. [Express information on the epidemic situation of HIV infection as of 01.01.2020 in the Irkutsk Region]. Available from: https://aids38.ru/?page_id=35.

2. Leshchenko OYa, Genich EV. [Reproductive disorders and their pathogenetic mechanisms in women with HIV]. HIV Infection and Immunosuppressive Disorders. 2019;11(4):20-29. doi: 10.22328/2077-9828-2019-11-4-20-29. [Article in Russian]. 3. Shugaeva SN, Savilov ED, Koshkina OG, Suzdalnitskiy AE, Chemezova NN. [Features of epidemic process of tuberculosis in the territory with high prevalence of HIV infection]. Acta Biomedica Scientifica. 2019;4(5):73-78. doi: 10.29413/ABS.2019-4.5.12. [Article in Russian].

4. Bayanova TA, Kudryavtseva DP, Plotnikova YuK, Botvinkin AD. [The change in the incidence of some herpes virus infections in population with a high prevalence of HIV infection]. HIV Infection and Immunosuppressive Disorders. 2019;11(3):75-84. doi: 10.22328/2077-9828-2019-11-3-7584. [Article in Russian].

5. Bärnighausen T, Hosegood V, Timaeus IM, Newell ML. The socioeconomic determinants of HIV incidence: evidence from a longitudinal, population-based study in rural South Africa. AIDS. 2007 Nov;21 Suppl 7(Suppl 7):S29-38. doi: 10.1097/01.aids.0000300533.59483.95..

6. Harling G, Bärnighausen T. The role of partners' educational attainment in the association between HIV and education amongst women in seven sub-Saharan African countries. J Int AIDS Soc. 2016 Feb 19;19(1):20038. doi: 10.7448/IAS.19.1.20038.

7. Petrova AG. [Perinatal HIV infection]. Irkutsk: ISCST;2020. [Book in Russian].

8. Leonova ON, Stepanova EV, Belyakov NA. [Severe and comorbid conditions in HIV patients: An analysis of adverse outcomes]. HIV Infection and Immunosuppressive Disorders. 2017;9(1):55-64. doi: 10.22328/2077-9828-2017-9-1-55-64. [Article in Russian].

9. Yastrebova EB, Samarina AV, Fertikh EK, Gutova LV. [Pediatric problems of HIV infection and solutions in Saint Petersburg]. HIV Infection and Immunosuppressive Disorders. 2019;11(1):31-37. doi: 10.22328/2077-9828-2019-11-1-3137. [Article in Russian].

10. Kravchenko EN, Yakovleva OA, Kuklina LV. [Obstetric and perinatal outcomes of preterm labor women living with HIV]. HIV Infection and Immunosuppressive Disorders. 2019;11(3):16-22. doi: 10.22328/2077-9828-2019-11-3-1622. [Article in Russian].

11. Balashova TN, Isurina GL, Skitnevskaya LV, Bard D, Tsvetkova LA, Volkova EN, et al. Study of alcohol consumption by pregnant and non-pregnant women in Russia. Acta Biomedica Scientifica. 2018;3(3):59-68. doi: 10.29413/
ABS.2018-3.3.9.

12. Pineles BL, Park E, Samet JM. Systematic review and meta-analysis of miscarriage and maternal exposure to tobacco smoke during pregnancy. Am J Epidemiol. 2014;179(7):807823. doi: 10.1093/aje/kwt334.

13. Shirley DK, Kaner RJ, Glesby MJ. Effects of smoking on non-AIDS-related morbidity in HIV-infected patients. Clin Infect Dis. 2013;57(2):275-282. doi: 10.1093/cid/cit207.

14. Chursina OA, Konstantinova OD, Kshnyaseva SK, Mazurovskaya OP. [Influence of tobacco smoking during pregnancy on the fetoplaceltal system and health of newborns]. Gynecology, Obstetrics and Perinatology. 2019;18(5):66-72. doi: 10.20953/1726-1678-2019-5-66-72. [Article in Russian]. 15. Savchenko GN, Koltsova OV. [Hardiness training for HIV-infected women]. HIV Infection and Immunosuppressive Disorders. 2020;12(3):111-119. doi: 10.22328/2077-98282020-12-3-111-119. [Article in Russian].

16. Maryanyan AYu, Slepchenko VV, Rashidova MA, Podkameneva TV, Kolesnikova LI. [Current ideas about the specific features of vaginal microbiocenosis in HIV-positive women of reproductive age]. Akusherstvo i Ginekologiya. 2019;(12):12-17. doi: 10.18565/aig.2019.12.12-17. [Article in Russian].

17. Gafurov YuT, Sundukov AV. [Clinico-laboratory specificities of the course of inflammatory diseases of the pelvic organs in HIV-infected patients]. Gynecology, Obstetrics and Perinatology. 2015;14(1):64-68. [Article in Russian].

18. Leshchenko OYa, Genich EV, Darenskaya MA, Kolesnikova LI. [HIV and infertility: Neuro-endocrine and metabolic aspects]. HIV Infection and Immunosuppressive Disorders. 2020;12(4):73-80. doi: 10.22328/2077-9828-202012-4-73-80. [Article in Russian].

19. Belyaeva E, Genich E, Leshchenko O. The Genotype Distribution of Human Papillomavirus among HIV-Infected Women Planning Pregnancy in Irkutsk, Russia. International Journal of Biomedicine. 2021;11(3):346-350. doi:10.21103/ Article11(3)_OA11.

20. Pembrey L, Newell ML, Tovo PA; EPHN Collaborators. The management of HCV infected pregnant women and their children European paediatric HCV network. J Hepatol. 2005;43(3):515-525. doi: 10.1016/j.jhep.2005.06.002.

21. Kolesnikova LI, Kolesnikov SI, Darenskaya MA, Grebenkina LA, Timofeeva E, Leshenko OYa, et al. Menstrual and reproductive function in women with $\mathrm{HIV}$-infection and antioxidant vitamins deficiency. J AIDS Clin Res. 2014;5(12):1-5. doi: 10.4172/2155-6113.1000382.

22. Mandelbrot L, Tubiana R, Le Chenedec J, Dollfus C, Faye A, Pannier E, et al. No perinatal HIV-1 transmission from women with effective antiretroviral therapy starting before conception. Clin Infect Dis. 2015;61(11):1715-1725. doi: $10.1093 / \mathrm{cid} / \operatorname{civ} 578$. 